\title{
Ion Signatures in Supernova Spectra
}

\author{
Kazuhito Hatano, David Branch, Adam Fisher, Jennifer Deaton and E. Baron \\ Department of Physics and Astronomy, University of Oklahoma, Norman, OK 73019; \\ hatano, branch, fisher, deaton, baron@mail.nhn.ou.edu
}

Received __; accepted _ 


\begin{abstract}
A systematic survey of ions that could be responsible for features in the optical spectra of supernovae is carried out. Six different compositions that could be encountered in supernovae are considered. For each composition, the LTE optical depth of one of the strongest optical lines of each ion is plotted against temperature. For each ion that can realistically be considered as a candidate to produce identifiable features in supernova spectra, a sample synthetic spectrum is displayed. The optical depth plots and the synthetic spectra can provide guidance to studies of line identifications in the optical spectra of all types of supernovae during their photospheric phases.
\end{abstract}

Subject headings: line: identification — supernovae: general 


\section{INTRODUCTION}

Owing to the strong Doppler broadening and line blending in supernova spectra, making line identifications can be difficult. Although many of the major features in the

optical spectra of supernovae have been identified, others still lack a secure identification. For example, some features that have occupied our attention recently are in the early spectra of the peculiar Type Ia SN 1991T (Fisher et al. 1998) and the early spectra of the peculiar Type Ic SN 1997ef (Deaton et al. 1998a). Our difficulty in identifying some of the features in the spectra of these and other supernovae has motivated us to carry out a systematic survey of the ions that could produce features in the optical spectra of supernovae. Shortly after the appearance of the Type II SN 1987A in the LMC, such a survey was published by Branch (1987), but only one composition was considered: hydrogen-rich, with a metallicity lower than solar by a factor of four. Here we consider six different compositions that could be encountered in supernovae.

In section 2, for each composition, LTE optical depths are calculated in the Sobolev approximation and plotted against temperature for one of the strongest optical lines of each ion. The LTE approximation has been shown to be useful for first-order interpretations of supernova spectra (e.g., Branch et al 1985; Jeffery \& Branch 1990; Harkness 1991a,b; Jeffery et al 1991, 1992; Filippenko et al. 1992a; Kirshner et al. 1993). The optical depth plots of section 2 show which ions can be considered as candidates for line identifications, and at which temperatures. Then a synthetic optical spectrum for each candidate ion is displayed in section 3. These plots show the spectral signatures of each of the candidate ions. By displaying sample synthetic spectra for all of the interesting ions in this paper, we will not have to repeat any of them in future papers based on SYNOW calculations (e.g. Fisher et al. 1998, Deaton et al. 1998a and 1998b). 


\section{LINE OPTICAL DEPTHS}

\subsection{Calculations}

In the Sobolev approximation (Sobolev 1960, Castor 1970, Jeffery \& Branch 1990), the optical depth of a line in a supernova that is expanding homologously with $v=r / t$ is

$$
\tau=\left(\frac{\pi e^{2}}{m c}\right) f \lambda t n_{l}\left(1-\left(\frac{g_{l} n_{u}}{g_{u} n_{l}}\right)\right)
$$

where $n_{l}$ and $n_{u}$ are the number densities in the lower and upper levels of the transition, $f$ is the oscillator strength, $t$ is the time since explosion, and the other symbols have their usual meanings. The Sobolev line optical depth is inversely proportional to the velocity gradient, i.e, proportional to $t$, because as a photon propagates it redshifts with respect to the matter; the larger the velocity gradient, the sooner the photon redshifts out of resonance with the transition, and the smaller the line optical depth. A convenient numerical form of equation (1) is

$$
\tau=0.026 f \lambda_{\mu} t_{d} n_{l}\left(1-\left(\frac{g_{l} n_{u}}{g_{u} n_{l}}\right)\right)
$$

where $\lambda_{\mu}$ is in microns, $t_{d}$ is in days, and $n_{l}$ is in $\mathrm{cm}^{-3}$.

In this paper we evaluate all line optical depths at the layer in the supernova where the electron scattering optical depth, $\tau_{e s}$, reaches unity. When electron scattering is the dominant opacity source, this layer can be thought of, roughly, as the bottom of the line-forming layer (although thermalization of the continuum will take place at a deeper layer or, in SNe Ia, perhaps not at all), and only transitions that achieve optical depths on the order of unity or greater at $\tau_{e s}=1$ will be able to form conspicuous features in the spectrum. We also will encounter situations where the electron scattering opacity will be 
smaller than the combined opacity of numerous lines. Then, effectively, the bottom of the line forming layer will be at a shallower layer in the supernova, and only lines that have optical depths well in excess of unity at $\tau_{e s}=1$ will be able to produce identifiable spectral features.

If the electron density is taken to decrease outward as a power law of index $n$, the electron density at $\tau_{e s}=1$ is given by

$$
n_{e}=(n-1) /\left(\sigma_{e} R\right)
$$

where $\sigma_{e}$ is the Thomson cross section and $R$ is the radius at which $\tau_{e s}=1$. We use $n=7$ and a characteristic value of $R=1.73 \times 10^{15} \mathrm{~cm}$, which corresponds to $v_{\text {phot }}=10,000 \mathrm{~km}$ $\mathrm{s}^{-1}$ and $t=20$ days. This gives $n_{e}=5.2 \times 10^{9} \mathrm{~cm}^{-3}$, which is used for all of the optical depth calculations of this paper. A value of $t=20$ days also is used in equation (2), except in section 2.7 where we discuss the time-dependent nickel-cobalt-iron composition that results from the radioactive decay of ${ }^{56} \mathrm{Ni}$ and ${ }^{56} \mathrm{Co}$. The use of constant characteristic values of $n_{e}$ and $t$ is sufficient for our purposes because the limiting approximation of this work is that of LTE.

Table 1 lists the relative abundances of the elements for five of the six compositions that we consider. The entries are logarithms of the abundance by number. Only the relative abundances are relevant; the absolute numbers have no significance. The way in which we arrived at each of these compositions, and where in supernovae they might be encountered, will be discussed below as the line optical depths are presented for each composition. (The sixth composition that we consider is the time-dependent mixture of nickel, cobalt, and iron that results from an initially pure ${ }^{56} \mathrm{Ni}$ composition.) Given the electron density and a composition, the Saha ionization and Boltzmann excitation equations are used to calculate the atomic level populations as well as the total gas density that is needed to provide 
the specified electron density. Figure 1, which shows the total density plotted against temperature for each of the six compositions, will be helpful in understanding the optical depth plots. As temperature falls, and significant donors of free electrons recombine, the total density rises in order to achieve the specified electron density. For example, for the solar composition, the density rise at $T \lesssim 6000 \mathrm{~K}$ is caused by hydrogen recombination, and for the helium-rich composition the steep density rise at $T \lesssim 10,000 \mathrm{~K}$ is caused by helium recombination.

For each ion, the optical depth of a reference line is calculated as a function of temperature. One of the ion's strongest lines in the range $4000<\lambda<10,000 \AA$ is chosen to be the reference line. Note that for O III and O II we are using forbidden lines as the reference lines, because they are stronger than any of the permitted lines in the temperature range considered here. The optical depth plots show the reference lines that achieve $\tau>0.001$ within the range $20,000>T>5,000 \mathrm{~K}$. These reference lines are listed in Table 2 , along with their $\log (g f)$ values and excitation potentials. The entry following the wavelength tells in which optical depth plots the ion makes an appearance; for example, 234 means that the ion appears on the optical depth plots for the second, third, and fourth compositions that we consider.

In this paper we make no allowance for nonthermal excitation and ionization. This is known to be significant for He I in the post-maximum spectra of Type Ib supernovae (Harkness et al. 1987; Lucy 1991) and may also be significant during certain phases of Type II (Jeffery \& Branch 1990) and Type Ic supernovae (Clocchiatti et al. 1997 and references therein; Deaton et al. 1998b). Such effects are discussed briefly below, when they may be relevant. 


\subsection{Hydrogen-rich}

The early spectra of Type II supernovae are formed in a hydrogen-rich composition, so we begin by considering the solar composition.

The optical depths are plotted in Figure 2. At high temperature no lines have $\tau>1$, and only $\mathrm{H} \alpha$ has $\tau>0.1$. As the temperature falls, $H \alpha$ becomes strong, followed by Ca II, Fe II, Ti II, and Sc II. Hydrogen is the main provider of free electrons throughout our temperature range, but at $T \simeq 6000 \mathrm{~K}$ it begins to become mostly neutral so the density must rise (Figure 1) to provide the specified electron density. This is the cause of the increase of the optical depths of singly ionized species such as Si II and Mg II at $T \lesssim 6000$ K. At these low temperatures, lines of many neutral species achieve $\tau>1$, as do the resonance lines of Sr II and Ba II.

Because hydrogen is the main source of free electrons, the effect of changing the metallicity is simple: the optical depths of all the heavy-element lines are proportional to the metallicity while the hydrogen and helium optical depths are unaffected. Lowering the hydrogen abundance in favor of helium would leave the hydrogen lines almost unaffected, because the total density would rise to give almost the same hydrogen density at the photosphere. The density rise would cause a strengthening of the metal lines, and helium lines would strengthen even more because of the increased helium abundance.

Most of the line optical depths are proportional to the square of the electron density. One factor of $n_{e}$ comes just from the associated increase in the total density, while the other comes from the Saha equation. This is because the lines of interest generally come not from the most abundant ion, but from the one that is once less ionized. For hydrogen, for example, increasing the specified electron density would not only increase the total

hydrogen density but also the fraction of hydrogen that is neutral, except for $T \lesssim 6000 \mathrm{~K}$ where most of the hydrogen already is neutral. 
The LTE optical depths of Figure 2 are, at least qualitatively, nicely consistent with the observed evolution of the spectra of Type II supernovae. Before and around the time of maximum light, SNe II are hot and their spectra are continuous except perhaps for weak hydrogen lines. As SNe II cool the hydrogen lines strengthen, followed by lines of Ca II, Fe II, Ti II and Sc II, as predicted. The most obvious discrepancy between prediction and observation is that the $\mathrm{Na}$ I D lines are calculated to be only about as strong as the reference lines of a number of other neutral species, whereas in the spectra of SNe II the feature produced by the D lines is considerably stronger than the lines of other neutrals. The answer, presumably, lies in non-LTE effects. A detailed study of line identifications in SN 1987A was carried out by Jeffery \& Branch (1990). In that particular case, too, the order of appearance of the lines was very much as discussed above. Lines that could be identified with confidence were from H, He I, Na I, Ca II, Sc II, Fe II, and Ba II. The very early appearance of a weak He I $\lambda 5876$ line possibly was due to an enhanced abundance of helium in the outer hydrogen-rich layers of SN 1987A, and the later (probable) appearance of the He I line is attributable to nonthermal excitation and ionization caused by the products of the radioactive decay of ${ }^{56} \mathrm{Co}$. The appearance of conspicous Ba II lines probably was due to an overabundance of barium (Mazzali \& Chugai 1995 and references therein).

The satisfactory agreement between calculated LTE optical depths and observation of SNe II suggests that the LTE optical depths presented below, for other compositions, also can be helpful for line identification studies.

\subsection{Helium-Rich}

The surface layers of SNe Ib are hydrogen-deficient and helium-rich, as may be the surface layers of SNe Ic. Such a composition also will be present, of course, beneath the outer hydrogen-rich layers of SNe II. We consider a helium-rich composition that is 
obtained by "burning" all of the hydrogen to helium. This increases the helium number abundance, relative to the heavier elements, by a factor of 3.55. (We make no allowance for conversion of carbon and oxygen into nitrogen by means of the CNO cycle.)

As can be seen in Figure 1, the density at the photosphere is higher in the helium-rich case than in the hydrogen-rich case, by a factor of about 3.1 when helium is singly ionized $(T \gtrsim 10,000 \mathrm{~K})$, but by a factor about 600 at $T \simeq 7500 \mathrm{~K}$, where helium is neutral. Figure 3 shows that at low temperature, oxygen, and then carbon, become the main sources of free electrons.

The line optical depths are shown in Figure 4. Apart from the absence of the hydrogen line and the strengthening of He I and He II due to the abundance change, the differences between the optical depths in the helium-rich and hydrogen-rich cases just reflect the difference in the density at $\tau_{e s}=1$, as a function of temperature. At high temperature no lines achieve $\tau>1$, and the He I line just makes it at $T \simeq 8000 \mathrm{~K}$. Considering the limitations of the LTE approximation, this means that the absence of conspicuous He I lines, at any temperature, is not evidence against a helium-rich composition. For $T \lesssim 10,000 \mathrm{~K}$, helium begins to recombine, the density rises sharply, the lines of heavy elements become much stronger at $\tau_{e s}=1$ than they were in the hydrogen-rich case, and line opacity dominates over electron scattering.

As is well known, the strong He I lines that are observed in the post-maximum light photospheric phases of SNe Ib require nonthermal excitation and ionization. Because the nonthermal effects increase the ionization of helium, whenever they occur they will cause the sharp density rise and the corresponding strengthening of the heavy element lines to shift to lower temperature than seen in Figure 4. 


\subsection{Carbon/Oxygen-Rich}

The outermost layers of SNe Ia and SNe Ic may be carbon/oxygen-rich. For example, in the model W7 (Nomoto, Thielemann, \& Yokoi 1984) the composition is C/O-rich down to $14,900 \mathrm{~km} \mathrm{~s}^{-1}$. We consider a composition that is obtained by burning all of the helium into equal amounts of carbon and oxygen by mass. This raises the carbon and oxygen number abundances, relative to the heavy elements, by factors of 147 and 52, respectively, and makes carbon more abundant than oxygen by a factor of 1.3 .

Carbon and oxygen are the principal sources of free electrons. Figure 1 shows that the density does not vary strongly with temperature. For $T \gtrsim 10,000 \mathrm{~K}$ the density is somewhat higher than in the hydrogen and helium rich cases, but the density does not increase sharply at low temperature because carbon remains partially ionized down to $5000 \mathrm{~K}$.

The line optical depths are shown in Figure 5. Now, for the first time, we see a line having $\tau>1$ at high temperature — that of C III — and at intermediate temperature the C II line has $\tau>1$. The forbidden reference lines of [O III] and [O II] have $\tau>0.1$. At low temperature, the O I and C I lines are only about as strong as they were in the helium-rich case, because in both cases the free electrons are mainly provided by carbon and oxygen. This means that, at least in LTE, the strength of the O I $\lambda 7773$ line cannot discriminate between a helium-rich and a $\mathrm{C} / \mathrm{O}$-rich composition. At low temperature the lines of elements heavier than oxygen are stronger than they were in the hydrogen-rich case, but not nearly as strong as they were in the helium-rich case, because their abundances relative to the main providers of free electrons, carbon and oxygen, are now reduced. 


\subsection{Carbon-burned}

Layers in which carbon has burned are expected to be encountered in the surface or subsurface layers of SNe Ia, and they are exposed above the photosphere before the time of maximum light. In model W7, the carbon-burned composition extends from 14,900 to about $13,000 \mathrm{~km} \mathrm{~s}^{-1}$. For a carbon-burned zone we consider composition number 5 from Table 3 of Khokhlov, Müller, \& Höflich (1993). Oxygen is the most abundant element, followed by silicon, sulfur, and magnesium. Oxygen is the main source of free electrons, except for $T \lesssim 6000 \mathrm{~K}$ where it recombines and silicon takes over. Figure 1 shows that the density runs somewhat higher than that of the $\mathrm{C} / \mathrm{O}-$ rich composition.

The optical depths are plotted in Figure 6. At high and intermediate temperatures, lines of silicon and sulfur ions are strong, and it is interesting that lines of P III and P II also have $\tau>1$. At $T \lesssim 8000 \mathrm{~K}$ lines of Si II, Ca II, and Mg II become very strong, and at $T \lesssim 7000 \mathrm{~K}$ lines of additional singly ionized and some neutral species also become strong. The O I line does not get much stronger than it was in the helium-rich and C/O-rich cases, so again the O I $\lambda 7773$ line is not a good composition indicator.

\subsection{Oxygen-burned}

In model W7 a layer in which oxygen has burned extends from about 9000 to 13,000

$\mathrm{km} \mathrm{s}^{-1}$. We consider composition number 4 from Table 3 of Khokhlov et al. (1993). The most abundant elements are silicon, sulfur, iron, argon, and calcium. Silicon is the main source of free electrons, and the density runs somewhat higher than in the carbon-burned case (Figure 1).

The optical depths are plotted in Figure 7. The main qualitative differences between these plots and those of the carbon-burned case are the disappearance of the oxygen and 
phosphorus lines, the weakening of Mg II, and the strengthening of Ca II, Fe III, and Fe II.

\subsection{Nickel-decay}

The sixth composition that we consider is the time dependent mixture of nickel, cobalt, and iron that results from the decay of ${ }^{56} \mathrm{Ni}$ through ${ }^{56} \mathrm{Co}$ to stable ${ }^{56} \mathrm{Fe}$. This composition has been suggested to dominate the outermost layers of the peculiar Type Ia SN 1991T (Filippenko et al. 1992b, Ruiz-Lapuente et al. 1992), or at least to be an significant component of those layers (Jeffery et al. 1992, Mazzali et al. 1995, Fisher et al. 1998), and large amounts of ${ }^{56} \mathrm{Ni}$ are present beneath the oxygen-burned zone in normal SNe Ia. Figure 8 shows the time dependent fractional abundances of nickel, cobalt, and iron, assuming an initially pure ${ }^{56} \mathrm{Ni}$ composition and using half lives of 6 and 77 days for ${ }^{56} \mathrm{Ni}$ and ${ }^{56} \mathrm{Co}$, respectively.

The density plotted in Figure 1 is for $t=20$ days. It is only a very weak function of the time, owing to the similar ionization potentials of nickel, cobalt, and iron. The density runs somewhat higher than in the oxygen-burned case, and it does not vary strongly with temperature.

Figure 9 shows the line optical depths for three different times: $t=5$ days, when the

fractional concentrations of nickel, cobalt, and iron are $0.55,0.45$, and $7 \times 10^{-3}$, respectively; at $t=20$ days $(0.09,0.81,0.1)$; and $t=80$ days $\left(7 \times 10^{-5}, 0.53,0.47\right)$. At 5 and 20 days, lines of the doubly or (depending on temperature) singly ionized species of all three elements are candidates for producing spectral features. At 80 days nickel lines are no longer a consideration. 


\section{SYNTHETIC SPECTRA}

In Figure 10 a sample synthetic spectrum is displayed for every ion that we consider to be a realistic candidate for producing an individually recognizable feature in the optical spectrum of a supernova. Not every ion that is listed in Table 2 appears in Figure 10. An ion such as V I, for example, that appears on an optical depth plot only in situations where numerous other ions are much stronger, is not a realistic candidate for producing identifiable spectral features.

Although the reference lines were selected from lines that have $\lambda<10,000 \AA$, the synthetic spectra of Figure 10 extend to $12,000 \AA$. The purpose of this is to show the feature produced by $\lambda 10830$ of He I, as well as features produced by other species such as Si I, which might produce the near infrared absorption feature that was observed in the spectrum of the Type Ic SN 1994I by Filippenko (1995). A discussion of the identification of this feature is presented by Deaton et al. (1998b).

The synthetic spectra are calculated with the parameterized supernova spectrum synthesis code SYNOW, which is an improved version of the code that has been used by in the past by, for example, Branch et al. (1985) and Filippenko et al. (1992a). The current version of SYNOW is described briefly by Fisher et al. (1997) and in detail by Fisher (1999). For the purposes of this spectrum atlas, we use the following parameters: the temperature of the blackbody continuum is $10,000 \mathrm{~K}$; the line optical depths decrease outwards as a power law of index 7; the velocity at the photosphere is $5000 \mathrm{~km} \mathrm{~s}^{-1}$; and the line forming region is truncated on the outside at a velocity of $30,000 \mathrm{~km} \mathrm{~s}^{-1}$. The optical depth of the reference line at the photosphere ordinarily is taken to be 10, but for some ions it is raised or lowered in order to better illustrate the spectral signature of the ion. Different excitation temperatures are used for different ionization stages because, for

example, lines of most neutral species have no chance of being seen unless the temperature 
is low. The excitation temperature is taken to be $5000 \mathrm{~K}$ for neutral species; $10,000 \mathrm{~K}$ for singly ionized; $15,000 \mathrm{~K}$ for doubly ionized; and 20,000 K for triply ionized.

Our intention here has been to show a synthetic spectrum for every ion that can realistically be considered as a candidate for producing an identifiable feature in the optical spectra of supernovae. Consequently the optical depth plots and synthetic spectra displayed in this paper can be used as a guide to line identifications in the optical spectra of all types of supernovae in their photospheric phases.

Figures and electronic versions of figures 10 may be obtained at: http://www.nhn.ou.edu/ baron/papers.htm

This work has been supported by NSF grants AST 9417102, 9417242, and 9731450. 


\section{REFERENCES}

Branch, D. 1987, ApJ, 320, L121

Branch, D., Doggett, J. B., Nomoto, K., \& Thielemann, F.-K. 1985, ApJ, 294, 619

Castor, J. I. 1970, MNRAS, 149, 111

Clocchiatti, A., et al. 1997, ApJ, 483, 675

Deaton, J., Branch, D., Fisher, A., \& Baron, E. 1998a, in preparation

Deaton, J. et al. 1998b, in preparation

Filippenko, A. V., et al. 1992a, AJ, 104, 1543

Filippenko, A. V., et al. 1992b, ApJ, 384, L15

Filippenko, A. V., et al. 1995, ApJ, 450, L11

Fisher, A. 1999, Ph.D. thesis, University of Oklahoma

Fisher, A., Branch, D., Nugent, P., \& Baron, E. 1997, ApJ, 481, L89

Fisher, A., Branch, D., Hatano, K., \& Baron, E. 1998, MNRAS submitted

Harkness, R. P. 1991a, in Supernovae, ed. S. E. Woosley (New York, Springer), 454

Harkness, R. P. 1991b, in SN1987A and Other Supernovae, ed. I. J. Danziger \& K. Kjär (Garching, ESO), 447

Harkness, R. P., et al. 1987, ApJ, 317, 355

Jeffery, D. J., Leibundgut, B., Kirshner, R. P., Benetti, S., Branch, D., \& Sonneborn, G. 1992, ApJ, 397, 304

Jeffery, D. J., \& Branch, D. 1990, in Jerusalem Winter School for Theoretical Physics, Vol. 6, Supernovae, ed. J. C. Wheeler, T. Piran, \& S. Weinberg (Singapore, World Scientific), 90 
Jeffery, D. J., Branch, D., Filippenko, A. V., \& Nomoto, K. 1991, ApJ, 377, L89

Khokhlov, A. J., Müller, E., \& Höflich, P. 1993, A\&A, 270, 223

Kirshner, R. P., et al. 1993, ApJ, 415, 589

Lucy, L. B. 1991, ApJ, 383, 308

Mazzali, P. A., \& Chugai, N. 1995, A\&A, 303, 118

Mazzali, P. A., Danziger, I. J., \& Turatto, M. 1995, A\&A, 297, 509

Nomoto, K., Thielemann, F.-K., \& Yokoi, K. 1984, ApJ, 286, 644

Ruiz-Lapuente, P., et al. 1992, ApJ, 387, L33

Sobolev, V. V. 1960, Moving Envelopes of Stars (Cambridge, Harvard University) 
Fig. 1. - The $\log$ of the total density $\left(\mathrm{gm} \mathrm{cm}^{-3}\right)$ at $\tau_{e s}=1$ is plotted against temperature for six different compositions.

Fig. 2.- The log of the Sobolev LTE optical depth of ion reference lines, evaluated at $\tau_{e s}=1$, is plotted against temperature for the hydrogen-rich composition.

Fig. 3.- The fractional contribution of free electrons is plotted against temperature for the helium-rich composition.

Fig. 4.- The log of the Sobolev LTE optical depth of ion reference lines, evaluated at $\tau_{e s}=1$, is plotted against temperature for the helium-rich composition.

Fig. 5.- The log of the Sobolev LTE optical depth of ion reference lines, evaluated at $\tau_{e s}=1$, is plotted against temperature for the $\mathrm{C} / \mathrm{O}$-rich composition.

Fig. 6. - The log of the Sobolev LTE optical depth of ion reference lines, evaluated at $\tau_{e s}=1$, is plotted against temperature for the carbon-burned composition.

Fig. 7. - The $\log$ of the Sobolev LTE optical depth of ion reference lines, evaluated at $\tau_{e s}=1$, is plotted against temperature for the oxygen-burned composition.

Fig. 8. - The fractional composition is plotted against time for the ${ }^{56} \mathrm{Ni}$-decay composition.

Fig. 9.- The log of the Sobolev LTE optical depth of ion reference lines, evaluated at $\tau_{e s}=1$, is plotted against temperature for the ${ }^{56} \mathrm{Ni}$-decay composition at times of 5,20 , and 80 days.

Fig. 10.- A synthetic spectrum is shown for each ion that can be regarded as a candidate for producing identifiable features in the photospheric-phase spectra of supernovae. 
TABLE 1

Atomic Abundances

\begin{tabular}{lcccccc}
\hline \hline element & atomic weight & H-rich & He-rich & C/O-rich & C-burned & O-burned \\
\hline $\mathrm{H}$ & 1.0 & 12.00 & - & - & - & - \\
$\mathrm{He}$ & 4.0 & 10.99 & 11.54 & - & 1.90 & 1.85 \\
$\mathrm{Li}$ & 7.0 & 1.16 & 1.16 & 1.16 & - & - \\
$\mathrm{Be}$ & 9.0 & 1.15 & 1.15 & 1.15 & - & - \\
$\mathrm{B}$ & 11.0 & 2.60 & 2.60 & 2.60 & - & - \\
$\mathrm{C}$ & 12.0 & 8.60 & 8.60 & 10.77 & 9.02 & 6.43 \\
$\mathrm{~N}$ & 14.0 & 8.00 & 8.00 & 8.00 & - & - \\
$\mathrm{O}$ & 16.0 & 8.93 & 8.93 & 10.65 & 11.84 & 7.45 \\
$\mathrm{~F}$ & 18.0 & 4.56 & 4.56 & 4.56 & - & - \\
$\mathrm{Ne}$ & 20.0 & 8.09 & 8.09 & 8.09 & 8.11 & 3.91 \\
$\mathrm{Na}$ & 23.0 & 6.33 & 6.33 & 6.33 & 7.52 & 4.10 \\
$\mathrm{Mg}$ & 24.0 & 7.58 & 7.58 & 7.58 & 10.81 & 7.22 \\
$\mathrm{Al}$ & 27.0 & 6.47 & 6.47 & 6.47 & - & - \\
$\mathrm{Si}$ & 28.0 & 7.55 & 7.55 & 7.55 & 11.18 & 11.76 \\
$\mathrm{P}$ & 31.0 & 5.45 & 5.45 & 5.45 & 9.44 & 6.23 \\
$\mathrm{~S}$ & 32.0 & 7.21 & 7.21 & 7.21 & 10.84 & 11.45 \\
$\mathrm{Cl}$ & 35.0 & 5.50 & 5.50 & 5.50 & 8.41 & 7.00 \\
$\mathrm{Ar}$ & 36.1 & 6.56 & 6.56 & 6.56 & 9.84 & 10.62 \\
$\mathrm{~K}$ & 39.0 & 5.12 & 5.12 & 5.12 & 7.17 & 6.87 \\
$\mathrm{Ca}$ & 40.0 & 6.36 & 6.36 & 6.36 & 8.28 & 10.44 \\
$\mathrm{Sc}$ & 44.5 & 3.10 & 3.10 & 3.10 & 5.94 & 5.60 \\
$\mathrm{Ti}$ & 45.0 & 4.99 & 4.99 & 4.99 & 7.95 & 8.30 \\
$\mathrm{~V}$ & 48.5 & 4.00 & 4.00 & 4.00 & 7.46 & 7.97 \\
$\mathrm{Cr}$ & 50.5 & 5.67 & 5.67 & 5.67 & 7.81 & 9.52 \\
$\mathrm{Mn}$ & 52.8 & 5.39 & 5.39 & 5.39 & 6.47 & 9.31 \\
$\mathrm{Fe}$ & 54.6 & 7.67 & 7.67 & 7.67 & 8.49 & 10.86 \\
$\mathrm{Co}$ & 56.0 & 4.92 & 4.92 & 4.92 & 8.65 & 6.29 \\
$\mathrm{Ni}$ & 56.8 & 6.52 & 6.52 & 6.52 & 8.80 & 9.53 \\
$\mathrm{Sr}$ & 87.6 & 3.02 & 3.02 & 3.02 & - & - \\
$\mathrm{Ba}$ & 137.3 & 2.10 & 2.10 & 2.10 & - & - \\
\hline & & & & & & \\
\hline
\end{tabular}


TABLE 2

REFERENCE LINES

\begin{tabular}{|c|c|c|c|c|c|c|c|c|c|}
\hline ion & $\lambda$ & & $\log (g f)$ & $\chi(\mathrm{eV})$ & ion & $\lambda$ & & $\log (g f)$ & $\chi(\mathrm{eV})$ \\
\hline H I & 6563 & 1 & 0.71 & 10.21 & K I & 7665 & 12345 & 0.13 & 0.00 \\
\hline He I & 5876 & 12 & 0.41 & 20.99 & $\mathrm{Ca} I$ & 4227 & 12345 & 0.26 & 0.00 \\
\hline He II & 4686 & 12 & 1.18 & 48.43 & Ca II & 3934 & 12345 & 0.15 & 0.00 \\
\hline C I & 9095 & 1234 & 0.07 & 7.49 & Sc I & 4024 & 1245 & 0.41 & 0.02 \\
\hline C II & 4267 & 1234 & 0.77 & 18.07 & Sc II & 4247 & 12345 & 0.32 & 0.32 \\
\hline C III & 4647 & 1234 & 0.08 & 29.57 & Ti I & 4533 & 12345 & 0.55 & 0.85 \\
\hline C IV & 5801 & 3 & -0.20 & 37.60 & Ti II & 4550 & 12345 & -0.45 & 1.58 \\
\hline N I & 8680 & 123 & 0.24 & 10.35 & Ti IV & 5399 & 45 & 0.16 & 26.37 \\
\hline N II & 5680 & 123 & 0.28 & 18.51 & V I & 4379 & 12345 & 0.59 & 0.30 \\
\hline N III & 4097 & 123 & -0.02 & 27.47 & V II & 4006 & 12345 & -0.76 & 1.82 \\
\hline O I & 7772 & 12345 & 0.32 & 9.16 & V III & 4070 & 45 & -2.04 & 7.86 \\
\hline$\left[\begin{array}{lll}\mathrm{O} & \mathrm{II}\end{array}\right]$ & 7321 & 234 & -8.43 & 3.33 & Cr I & 4254 & 12345 & -0.11 & 0.00 \\
\hline$\left[\begin{array}{lll}\mathrm{O} & \mathrm{III}\end{array}\right]$ & 4363 & 234 & -8.34 & 2.52 & Cr II & 4242 & 12345 & -0.59 & 3.87 \\
\hline $\mathrm{Ne} I$ & 6402 & 23 & 0.36 & 16.64 & Cr III & 5640 & 45 & -2.12 & 10.47 \\
\hline $\mathrm{Na} \mathrm{I}$ & 5890 & 1234 & 0.12 & 0.00 & Cr IV & 5940 & 5 & -1.97 & 19.29 \\
\hline Mg I & 5184 & 12345 & -0.18 & 2.72 & Mn I & 4031 & 12345 & -0.44 & 0.00 \\
\hline Mg II & 4481 & 12345 & 0.74 & 8.87 & Mn II & 4205 & 12345 & -3.58 & 1.81 \\
\hline $\mathrm{Al} \mathrm{I}$ & 8774 & 12 & -0.02 & 4.02 & Mn III & 4470 & 5 & -2.18 & 11.76 \\
\hline $\mathrm{Al} \mathrm{II}$ & 7042 & 123 & 0.35 & 11.33 & Mn IV & 4238 & 5 & -2.32 & 18.96 \\
\hline $\mathrm{Al}$ III & 5697 & 123 & 0.23 & 15.66 & $\mathrm{Fe} I$ & 4046 & 123456 & 0.28 & 1.48 \\
\hline Si I & 7944 & 1245 & -0.38 & 5.98 & Fe II & 5018 & 123456 & -1.40 & 2.89 \\
\hline Si II & 6347 & 12345 & 0.30 & 8.13 & Fe III & 4420 & 123456 & -2.22 & 8.24 \\
\hline Si III & 4553 & 12345 & 0.18 & 19.04 & $\mathrm{Fe} \mathrm{IV}$ & 4005 & 23456 & -1.87 & 23.64 \\
\hline Si IV & 4089 & 12345 & 0.20 & 24.08 & Co I & 4121 & 12456 & -0.60 & 0.92 \\
\hline P I & 9797 & 124 & 0.22 & 6.99 & Co II & 4161 & 123456 & -1.83 & 3.41 \\
\hline P II & 6508 & 234 & 0.62 & 10.90 & Co III & 4433 & 46 & -2.36 & 10.41 \\
\hline P III & 4222 & 34 & 0.21 & 14.63 & Co IV & 4246 & 6 & -4.20 & 16.48 \\
\hline P IV & 4250 & 4 & 0.00 & 29.05 & $\mathrm{Ni} \mathrm{I}$ & 5477 & 123456 & -0.35 & 1.83 \\
\hline S I & 9213 & 12345 & 0.42 & 6.53 & $\mathrm{Ni}$ II & 4067 & 123456 & -1.84 & 4.03 \\
\hline S II & 5454 & 12345 & 0.56 & 13.69 & Ni III & 4613 & 456 & -2.89 & 12.15 \\
\hline S III & 4254 & 12345 & 0.40 & 18.27 & Ni IV & 4316 & 6 & -2.59 & 23.09 \\
\hline $\mathrm{Cl} \mathrm{I}$ & 8376 & 24 & 0.46 & 8.93 & Sr II & 4078 & 123 & 0.17 & 0.00 \\
\hline Cl II & 4795 & 245 & 0.46 & 13.39 & Ba II & 4554 & 123 & 0.19 & 0.00 \\
\hline Ar I & 8115 & 245 & 0.41 & 11.56 & & & & & \\
\hline Ar II & 4348 & 2345 & 0.47 & 16.64 & & & & & \\
\hline Ar III & 4171 & 45 & -2.21 & 22.43 & & & & & \\
\hline
\end{tabular}

